\title{
A Deep Analysis of Higher Education Cognitive and Psychological Learning Impact During Covid 19 Pandemic
}

\author{
K. Srinivas ${ }^{\mathrm{a}, 1}$, Saravanan Pitchai ${ }^{\mathrm{b}}$, Udayabhanu N P G Raju ${ }^{\mathrm{c}}$, Abhishek Kumar ${ }^{\mathrm{d}}$, \\ B. Muthu Kumaran ${ }^{\mathrm{e}}, \mathrm{K}$. Vengatesan ${ }^{\mathrm{f}}$ \\ ${ }^{a l}$ School of Computer Science and IT, JAIN (Deemed to be University), Bangalore, India \\ ${ }^{b}$ Department of Computer Science and Engineering, SRM Institute of Science and Technology, \\ Kattankulathur, India \\ ${ }^{c}$ Department of Computer Science and Engineering, Aditya College of Engineering, Surampalem, India \\ ${ }^{d}$ Department of Computer Science \& Engineering, Koneru Laksmaiah Education Foundation, Hyderabad, \\ India \\ ${ }^{e}$ Department of Electronics and Communication Engineering, SRM Institute of Science and Technology, \\ Kattankulathur, India \\ ${ }^{f}$ Department of Computer Engineering, Sanjivani College of Engineering, Kopargaon, $M H$, India
}

\begin{abstract}
Current scenario around the globe we can find that physical or face to face learning got a very big full stop for a long period of time. Virtual learning took its place, somewhat leaving behind both its positive and negative impact on the education sector. E-learning is playing a chief part in maintaining the decorum of education sector. The research and surveys found that young learners got many benefits through this type of education but also it is undeniable that it has negative aspects too, which needs to be solved. Mainly private higher education suffered less as compared to institutions in rural areas. This research proposes how to bring out the quality of output through e-learning for all the learners equally. It has become a challenge for private and government institutions to make this smart or virtual learning as the best integral part of educational system.
\end{abstract}

Keywords. Cognitive Computing, Phycological Learning, Animation, Online education, virtual learning, COVID-19

\section{Introduction}

Virtual or online learning can work as a salvation or lifeline for those who find obstacles in learning or getting the proper education. It is like raising a tide to lift up all the boats. The best instance of it can be seen at the hour of covid-19 phase. All the sectors of the economy suffered from the huge downfall across the globe. But one of the most important sectors, which decided the future of our youth: educational sector, started working soon after a little pause and that pause was removed by e-learning practice. Students across the globe started learning back via online mode. But the few facts cannot be ignored that in few areas how negative impact was left out.

\footnotetext{
${ }^{1}$ K.Vengatesan,Department of Computer Engineering, Sanjivani College of Engineering, Kopargaon Email: vengicse2005@gmail.com.
} 
It was like a dumbstruck especially for rural areas students. Those facts were indisputable that how a large number of students suffered with their studies and even dropout condition came in front of them.

Online learning is not the newest form of learning but it came in trend from the last few decades and now became more popular in this pandemic period. Positive and negative findings were surveyed for this practice, which are discussed in the below research.

Innovation will never put back a prominent instructor but innovation in the reach of great instructor is transformational. Because of the internet facility in today's world, we can take a step forward towards the advancement of technology. This bridge leads to the path of destination which is more expedient to travel on. Education is granted as a precinct which is immune to change; simultaneously it faces a predicament of efficiency. From the various researches, I got to know that whenever people think about education, still the frequent thought is of schools and universities. The veracity is that no one actually noticed the significance of online learning, till the time everything was going smooth. It is really true that virtual learning still lacks behind than offline classroom learning. Mind-set of orthodox people still believes that proper education can be received only through offline learning that is received through the medium of schools and universities. That is so true that medium of text book to give education is one of the best ways to gain knowledge but we can't ignore the digital pattern of learning. Learning can be found outside of the school also in the ways we approach towards the theoretical knowledge same way we can approach in practical way, like through museums, art galleries, exhibitions, etc. But is this sufficient or adequate? One thing we can easily notice in today's generation that interactive learning, fun learning can create more interest among children for leaning and even their remembering capability also increases.

\section{Research Methodology}

The above literature suggests that this topic of e-learning was always highly debatable but now it came into more lime light because of the pandemic time due to covid-19. We got to know both the positive and negative finding of this. After knowing the importance and of this type of learning, government also took few steps forward towards the betterment and we can see that in the new education policy issued by the government.

Some consequences are not immediately recognizable, but some are noticed and some of them will be noticed after a long period of time. Like, especially for those students who mostly require or need practical knowledge. After thinking of many strategies and after applying many hit and trial methods few steps and decisions were taken to apply online mode of learning for all students across the globe, but now if we specially talk about our India, we didn't get $100 \%$ of the positive results. But few things cannot be unseen like few private institutions or universities applied this mode of learning correctly and came out with the positive results. Even many students got the advancement in their education method. To carry on and discuss the above issues on learning methods the below findings can be considered. 
Positive Finding: We can observe that large numbers of students were satisfied with the e-learning technique. This approach had left the positive impact for now and even for future. From the traditional way of teaching, students found e-learning more interesting in especially in contrast to interaction with teachers and other students, availability of study materials, building up the confidence to speak, improved viewpoint of studying. Participation of the students increased in webinars and class activities, leaving the fear of speaking behind in front of all. The deeper understanding of concepts also took place because of the recorded videos.

Negative Finding: The education of a country is theoretical and is carried in the classrooms itself. The treatise or notebooks are and have always been the root for the educational purposes. With the revolutionary idea that is adopted in the education system, books are being replaced by online notes or PowerPoint presentations or video lectures. India, a land where about $65 \%$ of people lives in rural areas, which are mal constructed and lack the basic amenities like Internet services or transportation. Generally, the people living in rural areas do not have access to technologies and believes in old school method of working. The children living in such areas face great difficulty in walking hand in hand with the new educational pattern. The online education, though, has coped up with the pace of the students and has not let our precious time go in vain but it also comes with a huge price of our health problems. Problems like stress, eyes weakness, migraine etc. The continuous use of mobiles and laptops Decreases the concentration of children and directly harms their health. Another major problem came out is of dropouts, because of not proper availability of resources students took this step and it cannot be measured in exact amount.

One of the most negative finding was found and noticed (at the time of covid-19 period) is about the non-teaching staff as they can't earn though online education. This department from educational institutions suffered a lot in financial aspect. At this time government and higher education department should build up the coordination to bring out the best possible outcome, keeping in mind about teaching, non-teaching staffs and students. All will take time to adapt the changes that occurred but it has become the necessity for today and future.

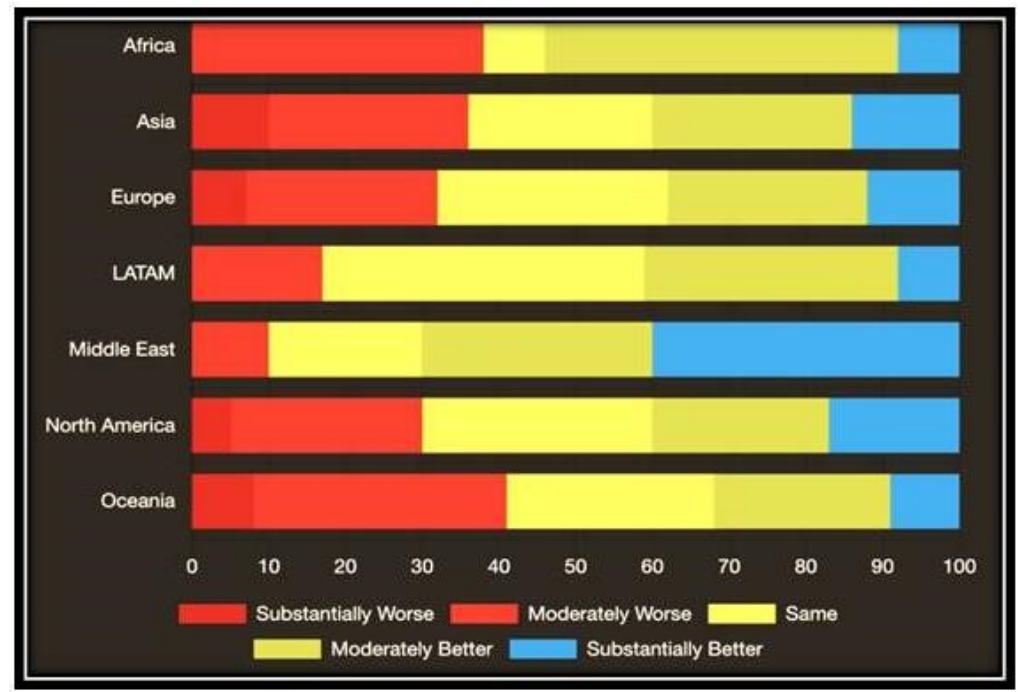

Figure 1. Graph Showing Expected Influence on Education Sector at The Time of Pandemic 
This graph shows the estimated impact on the educational sector globally. It also shows how positive and negative effects were occurred. If we focus on Asia part because of our country then we will find collision between moderately worst and moderately better. We can observe both the situations around us. Once we have a look at the rural side then we will find number of difficulties facing by students and teachers and on the other if we have a look at the urban side, then we will find some institutions gave a very positive result via an e-learning approach. Even the above methodology suggests the same that how educational sector got impacted in both positive and negative manner.

\section{Result and Analysis}

The below findings can help us to know more about this practice. There are many reasons to have discussion over e-learning, but some of them are very important to be noticed.

Effectiveness in learning: The quality of education improves when we not only focus on theoretical knowledge but also on practical knowledge. The way of online learning has become surprisingly increased and widespread learning approach in India due to the technologies.

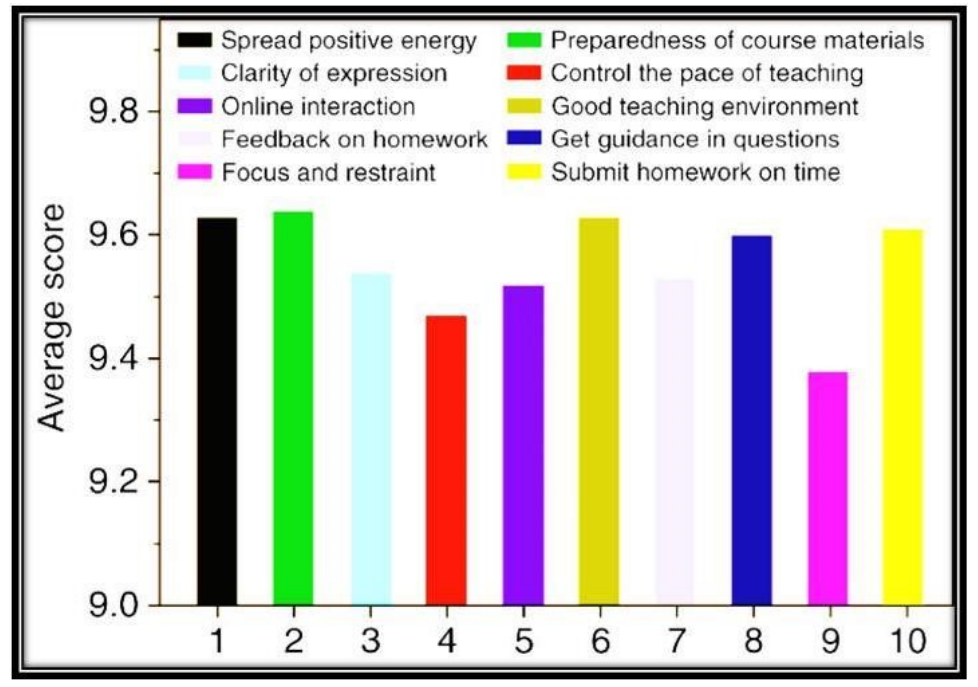

Figure 2. Coronavirus impact on e-learning

We can get the very clear view of how effective e-learning is, by seeing the growth of it at the time of pandemic (covid-19 period.) When there was no option left to maintain the proper decorum of studies then this process of learning was applied.

Networking moments: Because of e-learning students get the chance to develop their networks globally. They get the prospect to interact with different students across the nations and continents. This also helps them to become ethically strong and fit in the domain of others. 
Access to Proficiency: Students get the chance to learn from more pundit (expert) teachers or faculties. As even sometimes teachers can't come to a particular institution to teach students but in online that problem is also solved. Mainly here barriers due to geographical boundaries are removed.

Study materials: In e-learning our database is always more secured as compared to offline learning because we don't need to maintain files in cupboards and desks and there is always risk of losing that data. But for online learning we can keep our data secured in E-mails or in the online classroom applications, this helps to save the time of students to find the data and keep it for long period of time (as required by them). Accessibility to the documents increase.

Reduction in student's fees: Reduction of cost takes place as students don't need to pay transport, maintenance fees, etc. This helps to nurture the economic stability of the learner's family.

Location is never hindrance: We can study and learn from anywhere we just need a stable internet connection. For learning we don't need to travel from place to place. Even we don't need to go to buy study materials.

Revise or Emend Lectures straight away: Lectures can be revised at any time as they can be recorded. According to California psychologist students lose their focus five times in an around of forty-five minutes of lectures. So, in online students get the facility to rewind the audios and videos as many times as they please.

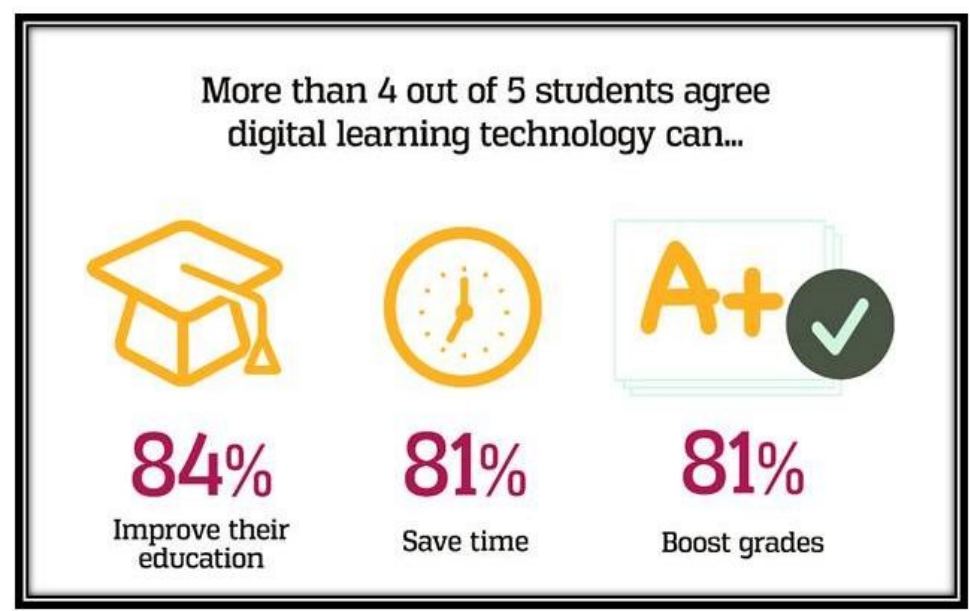

Figure 3. Net Survey analysis

This survey was mainly taken for college students but this is so true if we see around us. Mainly this image is shown as a reference over here just to show the positive impact on students because of online learning. These days only online learning is overcoming the offline method of teaching. The online method comes with lots of cons with it.

Problems in Rural Area: India is a country with about $65 \%$ of the population residing in rural areas where there is no source of electricity or Internet availability. A student of such areas faces a lot of daily problems to cope up with the studies to do online means. Many villages that do not have proper Internet or mean to access Internet hampers though education off the children. In many areas where the Internet is available the network connectivity issues become a hurdle in mode of learning. Due to 
net network connectivity the voice breakdowns or the video exposed which makes it difficult for students to study and even to concentrate. Due to lack of education in such areas the technical issues that arises is difficult to overcome. The student of junior classes faces a lot of problems as online means of education is not easy to comprehend for them and each individual does not get the attention they need. For such small children if patients are also illiterate then it becomes almost negligible for a student to focus. Students below eighth standard face a lot of problems in understanding and comprehending the topics that are taught in the class.

Problems in clearing the doubts: As a student It has many other drawbacks like the assignments given in the classes are difficult to complete as the in the classes many students are not able to clear their doubts which ultimately leads to pending assignments and increases the work load and pressure on student's mind. Even for the teachers it is difficult to teach as many teachers lacks behind in use of technology and faces problem to operate gadgets. Due to technical issues the time of the class gets wasted and there is only limited time slot given for each class.

Increase in the Workload: The workload of teachers has also increased as they have to make power point presentations or prepare video lecture in advance of all the topics. The mischievous students often try to disturb the class by not muting themselves and making random noises, they often remove their classmates from the classrooms or try to create other technical problems. Students who are good in computers and other technicalities disturb the class by finding the loopholes of the application and perform inappropriate actions.

Students lacking for teacher's attention: Another drawback that occurs is that teachers cannot pay proper attention to each student and taking advantage of this situation students leave the class unattended and leave or engage themselves in other activities. The doubts clearing session is also difficult to keep with the packed schedule of the teachers and unlike offline method a student individually cannot reach to the teacher and ask for the help.

Lack of Practical Sessions: With the rising online mode of teaching the field surveys cannot be performed and also the practical work, which needs to be done in workshops or outdoor, is failed to do so. The practical based knowledge can never be attained in the online method and we can never be able to know how to mold things or how to carry other mechanical works.

Examination mode: The online examination pattern is also not honestly given. When there is no one to keep an eye on the students they cheat and conduct their examination, with this way they always remain vague on topic and it is unfair for students who give examinations honestly and score the marks on their own capabilities. The objective type question or the multiple-choice questions can be found in the internet and so the subjective or theoretical type of questions. Even if the examinations are conducted via video mode, then also the network connectivity makes it difficult to keep an eye on the students. 


\section{COVID-19 Learning Loss: Reading forecast}

Forecasted trajoctories for grades 3-8, reading RIT scores based on COVID-19 induced school closures

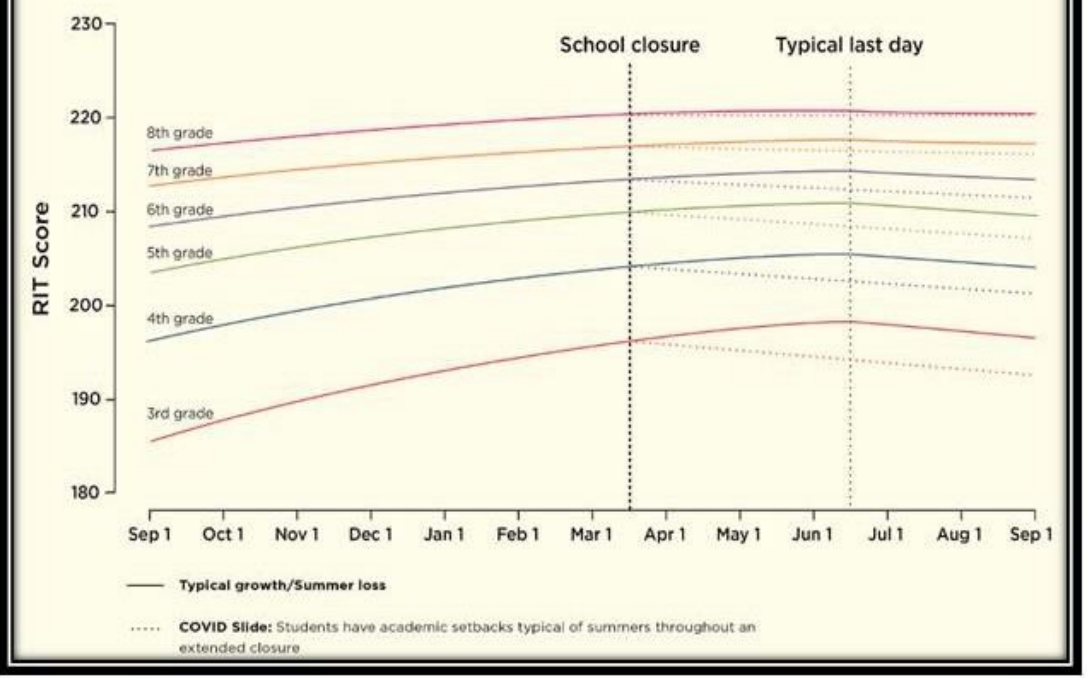

Figure 4: Impact of loss in student's education during pandemic

During the time of covid-19 time, students suffered lot because of hindrances which occurred in virtual learning. Especially for rural areas it was equal to classes going on or not.

\section{Conclusion and Future discussion}

Seeing the excitement and necessity for online learning in today's era it has become a vital part of educational zone. Furthermore, in offline learning, students interact with their faculties and peers to discuss the concepts or problems but they can't do same in online learning at the same level. But after seeing the both positive and negative sides of virtual learning and face to face learning, few steps are required to be taken. Uniqueness of both type of teachings should be found to bring out the finest results.

\section{References}

[1] S. Babić, S. K. Sučić and G. Sinković, "Understanding the Factors that Influence Secondary

[2] School Teachers' Intention to Use e-Learning Technologies for Teaching After the COVID19 Pandemic," 2020 43rd International Convention on Information, Communication and Electronic Technology (MIPRO), Opatija, Croatia, 2020, pp. 848-853.

[3] S. Dyubele, S. Soobramoney and D. Heukelman, "Using Smartphones as Constructivist Learning Tools," 2020 International Conference on Artificial Intelligence, Big Data, Computing and Data Communication Systems (icABCD), Durban, South Africa, 2020, pp. 1-8.

[4] S. Sharma and D. Verma, "Impact of COVID-19 and Nine-Minute Call on Indian Power Sector: Study of Blackout Prevention," 2020 International Conference on Technology and Policy in Energy and Electric Power (ICT-PEP), Bandung, Indonesia, 2020, pp. 301-305. 
[5] M. Tropea and F. De Rango, "COVID-19 in Italy: current state, impact and ICT-based solutions," in IET Smart Cities, vol. 2, no. 2, pp. 74-81, 72020.

[6] Q. Pham, D. C. Nguyen, T. Huynh-The, W. Hwang and P. N. Pathirana, "Artificial Intelligence (AI) and Big Data for Coronavirus (COVID-19) Pandemic: A Survey on the State-of-theArts," in IEEE Access, vol. 8, pp. 130820-130839, 2020.

[7] Kumar, A., Vengatesan, K., Rajesh, M., \& Singhal, A. (2019). Teaching literacy through animation \&amp; multimedia. International Journal of Innovative Technology and Exploring Engineering, (5), 73-76.

[8] A. Kumar, K. Vengatesan, A. Singhal and D. K. Verma, "3D Lighting Courseware development for 3D Motion Picture Science," 2018 International Conference on Recent Innovations in Electrical, Electronics \& Communication Engineering (ICRIEECE), 2018, pp. 2321-2323, doi: 10.1109/ICRIEECE44171.2018.9009258.

[9] A. Kumar, A. Singhal, K. Vengatesan and D. K. Verma, "Study and Research of 3D Animation Courseware Development," 2018 International Conference on Recent Innovations in Electrical, Electronics \& Communication Engineering (ICRIEECE), 2018, pp. 2514-2516, doi: 10.1109/ICRIEECE44171.2018.9008670.

[10] J. Adam Jones, J. Edward Swan II, Gurjot Singh, Josh Franck, and Stephen R. Ellis. The Effects of Continued Exposure to Medium Field Augmented and Virtual Reality on the Perception of Egocentric Depth. In Poster Compendium, Proceedings of ACM SIGGRAPH Symposium on Applied Perception in Graphics and Visualization (APGV 2009), pp. 138, September 2009. DOI: $10.1145 / 1620993.1621032$.

[11] M. N. Islam and A. K. M. N. Islam, "A Systematic Review of the Digital Interventions for Fighting COVID-19: The Bangladesh Perspective," in IEEE Access, vol. 8, pp. 114078114087, 2020.

[12] Potharaju, S. P., Sreedevi, M., \& Amiripalli, S. S. (2019). An Ensemble Feature Selection Framework of Sonar Targets Using Symmetrical Uncertainty and Multi-Layer Perceptron (SU-MLP). In Cognitive Informatics and Soft Computing (pp. 247-256). Springer, Singapore.

[13] D. D. N. Benty et al., "Use of Information and Communication Technology in Leaming in the Covid-19 Pandemic Period to Improve Student Learning Outcomes," 2020 6th International Conference on Education and Technology (ICET), Malang, 2020, pp. 165-169.

[14] A. K. Bairagi et al., "Controlling the Outbreak of COVID-19: A Noncooperative Game Perspective," in IEEE Access, vol. 8, pp. 215570-215581, 2020.

[15] M. Pokorný, "Experience with e-learning in Teaching Combinatorics and Data Processing," 2020 43rd International Convention on Information, Communication and Electronic Technology (MIPRO), Opatija, Croatia, 2020, pp. 838-842. 